\title{
INFLUENCE DES CONDITIONS DE COUPLAGE D'UN INTERFACE SUR LA FORME DU SIGNAL IMPULSIONNEL
}

\author{
Y. JAYET, J.C. BABOUX et M. PERDRIX \\ Laboratoire de Traitement du Signal et Ultrasons, INSA, Bât. 502, F-69621 Villeurbanne cedex, \\ France
}

\section{Summary}

Numerous theoretical studies have shown that the reflection and transmission phenomena of an ultrasonic plane wave can be expressed by complex coefficients depending on the acoustic properties of the different media and on the coupling conditions of the interface. In pulsed mode, the application of these complex coefficients changes the shape of the signal. Then, for a given incident shear wave, we study the influence of the quality of the interface, characterized by boundaries coefficients, on the shape of the reflected shear wave. So, we have proven that the quality of the interface cannot be determined only from the amplitude of the reflected signal but, we must consider its shape depending on the argument of reflection coefficient.

\section{I - Introduction}

Lorsqu'une onde plane arrive sur un interface plan séparant deux milieux, elle donne naissance à des phénomènes de reflexion et de transmission qui s'accompagnent en général de conversion de mode.

Des études théoriques effectuées en régime sinusoïdal ont abouti à exprimer analytiquement ces phénomènes à l'aide de coefficients qui deviennent complexes au delà d'une incidence supérieure au ler angle critique. L'application de ces coefficients complexes se traduit en régime impulsionnel par une modification de la forme des signaux réfléchis ou transmis.

Dans ce travail, nous caractérisons la qualité du couplage à l'interface par des coefficients représentatifs des relations de continuités. Par simulation numérique, nous avons alors mis en évidence que l'amplitude et la phase du coefficient de reflexion de l'onde transversale créée à l'interface sont fonction de ces conditions de couplage.

Ainsi, en considérant le cas de l'interface duralumin-résine représentatif d'une structure collée, et après avoir déterminé par simulation numérique l'angle d'incidence optimum, nous étudions expérimentalement la qualité du couplage en analysant la forme de l'onde transversale réfléchie. En effet, en régime impulsionnel 
l'analyse temporelle d'un signal déformé ne traduit pas seulement son évolution en amplitude mais prend en compte la phase.

\section{II - Détermination des coefficients de reflexion et de transmission}

Considérons deux milieux solides homogènes et isotropes délimités par une surface plane. L'onde plane uniforme sinusoïdale incidente rencontre l'interface sous un angle d'incidence $\mathrm{B}^{\mathrm{I}}$ et au contact de la discontinuité sont générées des ondes planes réfléchies et transmises de type longitudinale et transversale dont les directions de propagation sont liées aux vitesses par la loi de Snell.

Les amplitudes respectives de déplacement de ces ondes réfléchies et transmises sont déterminées à partir de l'amplitude de l'onde incidente en appliquant les conditions aux limites traduisant la continuité des composantes normales et tangentielles des déplacements et des contraintes.

Si l'angle d'incidence est inférieur au premier angle critique, les relations liant les différentes amplitudes sont réelles. Par contre, dès que l'angle d'incidence devient supérieur à ce premier angle critique, pour satisfaire la loi de Snell, nous sommes conduit à introduire des angles complexes qui rendent ces relations complexes.

Dans ces conditions, nous ferons intervenir des coefficients complexes de réflexion et de transmission définis par :

$$
\alpha=\operatorname{Ae}^{\varphi}
$$

ou A est le module et $\uparrow$ l'argument.

\section{III - Détermination de la qualité d'un interface}

\subsection{Etude théorique et simulation numérique}

La qualité de la liaison entre deux milieux séparés par un interface est exprimée analytiquement par les relations particulières existantes entre les composantes normales et tangentielles des déplacements et des contraintes.

Nous introduisons deux coefficients $k_{N}$ et $k_{T}$ qui caractérisent les discontinuités des composantes normale et tangentielle du déplacement. Par contre, comme d'autres auteurs [2,3], nous admettrons qu'il y a toujours continuité des composantes normale et tangentielle des contraintes. Dans ces conditions, l'amplitude et l'argument des coefficients de reflexion et de transmission calculés précédemment dépendent des valeurs de $\mathrm{k}_{\mathrm{N}}$ et $\mathrm{k}_{\mathrm{T}}$ donc de la qualité de la liaison à l'interface.

Dans ce travail, nous présentons deux cas limites; le couplage solide pour lequel $\mathrm{k}_{\mathrm{T}}=\mathrm{k}_{\mathrm{N}}=1$ et le couplage liquide symbolisé analytiquement par $\mathrm{k}_{\mathrm{T}}=0$ et $\mathbf{k}_{\mathrm{N}}=1$.

Pratiquement, les ondes transversales, de part leur nature sont les plus sensibles aux discontinuités de type solide-liquide. Par simulation numérique, nous avons donc tracé l'amplitude et l'argument du coefficient de reflexion en mode transversal dans le cas de l'interface duralumin-résine représentatif d'une structure collée et irradiée par une onde ultrasonore transversale. Les valeurs de l'amplitude et de l'argument sont représentées en fonction de l'angle d'incidence sur les courbes 
1-a dans le cas d'un couplage solide $\left(k_{T}=k_{N}=1\right)$ et sur les courbes $1 \mathrm{~b}$ dans le cas d'un couplage liquide $\left(\mathrm{k}_{\mathrm{T}}=0, \mathrm{k}_{\mathrm{N}}=1\right)$.
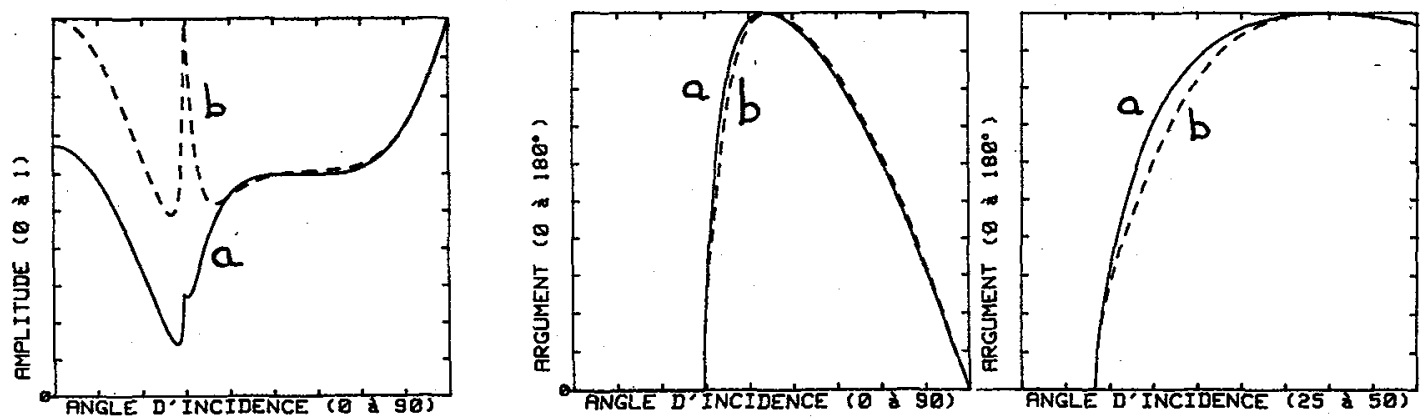

Figure 1: Amplitude et argument du coefficient de reflexion de l'onde transversale dans le cas de l'interface duralumin-résine : 1-a couplage solide ; 1-b couplage liquide.

\subsection{Application à la caractérisation de l'interface}

A partir des courbes précédentes, on constate que pour une incidence légèrement supérieure au ler angle critique $\left(29^{\circ}\right)$, nous avons selon la nature du couplage une forte variation de l'amplitude et de l'argument du coefficient de reflexion de l'onde transversale.

Des études précédentes ont considéré l'amplitude du signal réfléchi $[2,3]$ et ont relié le module du signal réfléchi à la qualité du couplage. Le but de notre étude est de prendre en compte la différence de phase du signal réfléchi ce qui se traduit en régime impulsionnel par une déformation du signal, analysée aisément par tout système numérique.

Expérimentalement, nous nous sommes placés à une incidence égale à $33^{\circ}$ à l'aide du montage suivant (figure 2).

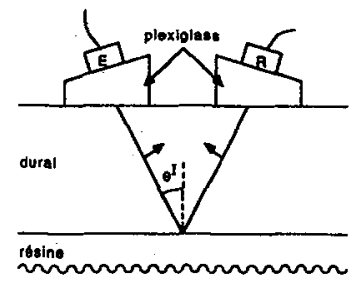

Figure 2 : Dispositif expérimental

La génération de l'onde ultrasonore incidente est assurée après transformation de mode par un traducteur longitudinal large bande. Le couplage solide est réalisé par collage de la résine sur un bloc de duralumin à faces parallèles alors que le couplage liquide est symbolisé par la présence d'un film d'huile entre les deux solides. 
Les courbes $3 a$ et $3 b$ représentent les signaux mesurés aux bornes du traducteur récepteur large bande après réflexion sur les deux interfaces étudiées et décrits précédemment.
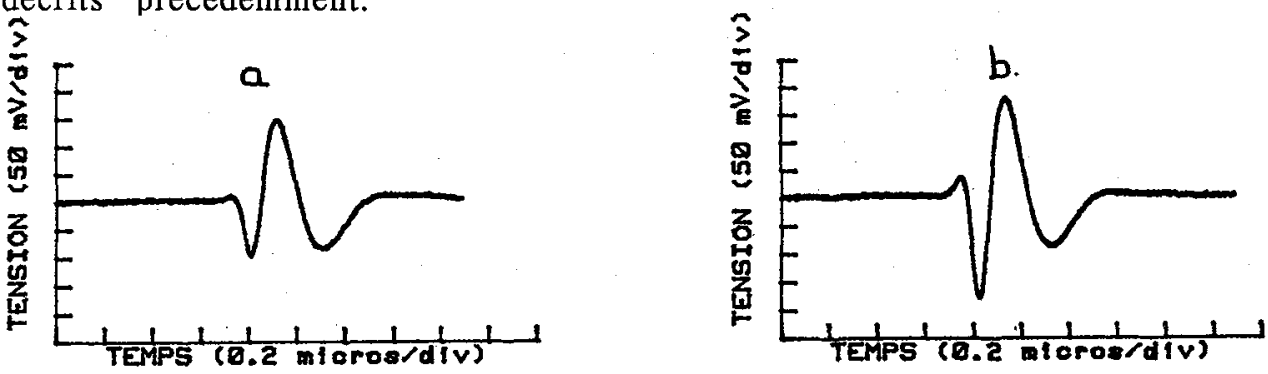

Figure 3 : Signaux ultrasonores après réflexion sur l'interface $a$ : couplage solide ; $b$ : couplage liquide

Par une observation visuelle, on constate que ces signaux sont déformés l'un par rapport à l'autre. Une analyse numérique plus approfondie utilisant le concept de signal analytique permet de quantifier la différence de phase causant cette déformation et nous mettons alors en évidence que le résultat est conforme aux prévisions théoriques de la figure $1\left(\Delta \varphi \simeq 20^{\circ}\right)$. D'autre part, au regard de ces deux signaux, qui d'après les courbes 1 sont d'amplitudes très différentes, nous constatons qu'en régime impulsionnel la comparaison en amplitude de deux signaux n'a de sens que s'ils sont de forme identique. Dans le cas contraire étudié ci-dessus, il faudrait alors effectuer une analyse fréquentielle de ces signaux et comparer les amplitudes de leurs spectres à une fréquence donnée.

\section{IV - Conclusion}

Nous avons mis en évidence que le phénomène de reflexion avec transformation de mode se traduit en régime impulsionnel par une déformation des signaux réfléchis qui dépend fortement des propriétés acoustiques des milieux mis en présence et des conditions de couplage de l'interface.

On peut alors envisager une technique basée sur ce principe pour contrôler la qualité des interfaces sachant qu'en régime impulsionnel, nous ne pouvons pas étudier les variations d'amplitude des signaux réfléchis sans prendre en considération leurs phases. Bien que plus délicate à mettre en oeuvre car nécessite un traitement numérique, cette voie de recherche portant sur l'analyse de l'argument du signal réfléchi doit être prise en compte et mérite d'être approfondie.

\section{Bibliographie}

1. W.G. Mayer - Energy partition of ultrasonic waves at flat boundaries, Ultrasonics, 1965 , p. 62-69.

2. Pilarski Rose - A transverse wave ultrasonic oblique incidence technique for interfacial weakness detection in adhesive bond, J. Appl. Phys., 63, 2, 1988, 300307.

3. Pilarski-Rose - Ultrasonic oblique incidence for improved sensitivity in interface weakness determination NDT International, 21, 4, 1988, 241-246. 